\title{
Estudio de la autopercepción del rendimiento académico de los estudiantes universitarios de primer curso
}

\author{
Larisa Dunai ${ }^{a}$, José Alfonso Antonino Daviu ${ }^{b}$, Ismael Lengua ${ }^{a}$ y Guillermo Peris- \\ Fajarnés $^{\text {a }}$ \\ ${ }^{a}$ Universitat Politècnica de Vàlencia, Department of Graphic Engineering, Valencia, 46022, Spain; \\ ${ }^{\mathrm{b}}$ Universitat Politècnica de Vàlencia, Department of Electrical Engineering, Valencia, 46022, Spain
}

\begin{abstract}
Resumen
El rendimiento academico es un factor importante en los logros academicos, motivación, etc. del estudiante y la Universidad. Los resultados academicos de los estudiantes como de la Universidad en si, se reflejan en las notas e indicadores de calidad. El rendimiento academico de los estudiantes no solo se refleja en la actitud del alumno, sino tambien en los resultados del profesorado y en la Universidad en si. Se ha realizado un estudio con 17 alumnos del primer curso. Los resultados han concluido que un $65 \%$ de los estudiantes consideran que sus logros academicos son sus logros personales, el 59\% considera que se preparan muy bien las asignaturas y un $41 \%$ son su respuesta es neutral con sus estudios. Solo un $47 \%$ de los estudiantes consideran que los métodos de estudio son adecuados y 29\% neutral. Un 50\% de los estudiantes tienen afianzados los conocimientos. Este hecho les provoca inseguridad, que es un factor importante en el rendimiento academico ya que de ella depende la motivación y la tranquilidad durante las pruebas evaluativas, etc.
\end{abstract}

Palabras clave: rendimiento académico, preparación de la asignatura, metodología de aprendizaje, conocimientos, estudiantes universitarios.

\footnotetext{
Abstract

The academic achievement is an important factor as the student and university academic success, motivation, etc. The student and university academic success conclude in an indicator/mark of academic success that represent the successfulness of the student and university. The academic performances of the students are not only reflected in the student attitude and its future, but also in the docent results of the university itself. 17 students from the firs university
} 
Estudio de la autopercepción del rendimiento académico de los estudiantes universitarios de primer curso

academic year participated voluntary in the test. Results define that $65 \%$ of the students consider that their academic achievements are their personnel achieve. Over 59\% consider that they prepare well the subjects and $41 \%$ are indifferent to the studies. Only $47 \%$ of the participants consider that their study methods are adecuadly, 29\% consider indifferent. Over $50 \%$ of the participants are unsure of their knowledge and capacities. The insecurity is one of the most important factor in academic performance, since it depends on the motivation, tranquility at the time of testing, etc.

Keywords: academic performances, subject preparing, study methods, insecurity on their abilities and knowledge, university students.

\section{Introducción}

El éxito del modelo educacional depende del logro académico. Se considera que el nivel social de un país depende en gran parte al nivel intelectual de su sociedad. Con este fin muchos países desarrollados hacen hincapié en la educación y formación profesional. Para evaluar el rendimiento académico de los estudiantes tanto escolares de los institutos como los estudiantes de universidad se realizan pruebas evaluatorias del conocimiento. Cabe mencionar que los estudiantes universitarios con alto rendimiento académico tienen más posibilidad de empleo y mejores salarios (Joppke \& Morawska, 2003).

El objetivo del estudio, es valorar algunos parámetros que puedan afectar al rendimiento académico. El rendimiento académico está influenciado por la motivación, autoestima y la inteligencia de cada estudiante. En la universidad llegan los mejores estudiantes, con una inteligencia alta y un alto grado de preparación. Sin embargo, en el primer año de carrera, hay una tasa de fracaso académico muy alta. ¿Cuál es la causa de dicho fallo? .

La inteligencia y la preparación no es suficiente para permanecer en la universidad y acabar con éxito la carrera elegida. Uno de los factores importantes para ello se debe a la motivación de cada alumno, el interés, esfuerzo y elegir correctamente la carrera. El deseo de realizar una carrera del propio estudiante o el deseo de la familia, así como sobrestimar sus capacidades puede llevar al fracaso escolar (Marsh, 1990). Es muy importante que cada estudiante antes de elegir la carrera, realice un estudio de capacidad para ella. Hay muchas formas de realizar dicha evaluación: hacer visitas a la universidad, asistir como oyente a algunas clases en las asignaturas básicas de la especialidad, investigar el rendimiento académico de la escuela y especialidad, hablar con los alumnos de la especialidad para tener conocimientos. 
La autopercepción tiene un rol importante en el rendimiento académico (Guay et al, 2003; Choi, 2005; Liu, 2005; Muijs, 1997). En líneas generales, los estudiantes sobrestiman sus habilidades sin tener en cuenta la dificultad de las tareas propuestas (Falchikov \& Bond, 1989). Red et al, (2001) define la autopercepción académica como la "percepción de los estudiantes de sus competencias y habilidades con respeto al ámbito académico," lo que confirma las afirmaciones de Lent et al (1997). Esto quiere decir que la autopercepción indica como los estudiantes se sienten consigo mismo, como se ven a sí mismos frente a la especialidad elegida. Thorpe, et al., en su estudio demostró que el 90\% de los alumnos del primer año en la educación superior se consideran estar en la media o por encima de ella. En su estudio Green et al., (2006) llegó a la conclusión que "mejorando el auto concepto académico y la motivación conduce a mejorar el rendimiento académico y un rendimiento académico mejorado conduce a incrementar el auto concepto y la motivación académica". March (1990) confirma que un alto autoconcepto académico está asociado a un mejor rendimiento académico. De aquí se puede deducir que la motivación, el auto concepto están relacionados con el rendimiento académico.

$\mathrm{Y}$ bien, para poder evaluar la motivación se hay que mencionar la existencia de tres tipos (Crous et al., 2000):

1. La motivación intrínseca se refiere a la acción que uno desarrolla porque quiere y no necesitan incentivos externos.

2. La motivación extrínseca se refiere a las acciones que se realizan con el fin de obtener una recompensa.

3. Amotivación ocurre cuando un estudiante no percibe contingencias entre sus acciones y sus resultados. La amotivación se define como no tener motivación.

El rendimiento académico indica el valor numérico del conocimiento de los estudiantes, así como mide la adaptación de los estudiantes a la especialidad, vida universitaria sistema de enseñanza universitaria, etc.

\section{Método}

El estudio han participado 17 alumnos de nuevo ingreso en la carrera de Grado en Ingeniería Industrial en el segundo semestre. Todos los alumnos están matriculados en la asignatura troncal Expresión Gráfica. Todos los estudiantes tienen un gran nivel de conocimiento previo ya que vienen con perfil técnico. Se considera que todos los participantes tienen habilidades y capacidades para cursar la carrera. Los alumnos has respondido a un cuestionario desarrollado por los investigadores, con el fin de definir el autoconcepto, la motivación y relacionarlo con el rendimiento académico. 
Estudio de la autopercepción del rendimiento académico de los estudiantes universitarios de primer curso

El objetivo del presente trabajo es determinar si la autopercepción y la motivación influye en el rendimiento académico de los estudiantes.

En la Tabla 1 se presentan los resultados obtenidos de la evaluación de la autopercepción de los estudiantes participantes en el estudio. Se han definido el promedio y la desviación estándar de la confianza y el esfuerzo.

Tabla 1. Promedio y la desviación estándar de los resultados en la autopercepción académica.

\begin{tabular}{|c|c|c|}
\hline Característica & Promedio, \% & Desviación Estándar \\
\hline $\mathrm{AP}$ & - & - \\
\hline Confianza & 46.1 & 6.9 \\
\hline Esfuerzo & 50.25 & 21.2 \\
\hline Estrés & 52.7 & 26.9 \\
\hline
\end{tabular}

De los $46,1 \%$ de los alumnos confiados en sí mismos un $65 \%$ consideran que sus logros es el resultado de sus propios esfuerzos, el $47 \%$ están muy seguros de ello y el $35 \%$ consideran que sus logros son el resultado de esfuerzos compartidos. De aquí se puede observar que solo el 35\% de los participantes en el estudio, aprecian el esfuerzo de los terceros como familiares, amigos, colegas y docentes. Debido a que la presencia a las clases es obligatoria en el grado en la Universitat Politècnica de València y la docencia es en mayoría docencia de clase magistral, donde el docente explica en detalles tantos teóricos como prácticos el contenido de la asignatura, se puede determinar que con tener el programa de la asignatura ellos son capaces de aprobar las asignaturas estudiando de forma individual y autónoma. Resulta que la labor del docente, se debe reenfocar para que las clases no sean magistrales si no de docencia inversa. De esta forma, durante las horas de clase, el docente realizará la labor de tutor, resolviendo dudas y hacer hincapié en los términos y modelos más significante de cada tema.

El 59\% de los participantes, han contestado que antes de ir a las pruebas evaluatorias se preparan bien el contenido de la asignatura. Del 59\% solo el 6\% están muy seguros de estar muy bien preparados para las pruebas evaluatorias. Un 52,7\% de los alumnos afirman estar estresados en las pruebas evaluatorias. Si analizamos bien los datos se puede observar que los estudiantes se estresan por causas más bien psicológicas, que por dominar la materia. $\mathrm{Al}$ preguntarles si se suelen sentir ansiosos durante el examen un 11,8\% están aterrorizados, un $47,1 \%$ están ansiosos, un 17,6 indiferentes y el resto están tranquilos. A la pregunta si al realizar evaluaciones les causa estrés, un $29,4 \%$ están muy de acuerdo y $41,2 \%$ están de acuerdo y el resto $29,4 \%$ son indiferentes. Analizando las dos preguntas observamos que el 
estrés causa ansiedad y la ansiedad conduce a malos resultados de rendimiento académico. Se puede deducir que la motivación de los estudiantes depende de la confianza y la autoestima. Si un estudiante nada más pensar en las pruebas evaluativas se estresa, resulta que su nivel de motivación y confianza se reduce.

Por otro lado, los hábitos de estudio representan un impacto muy significativo en el rendimiento académico. Un 58,8\% afirma que no dedican suficiente tiempo a los estudios y un $29,4 \%$ afirma que estudia la noche anterior al examen y un $23,5 \%$ le es indiferente.

\section{Conclusiones}

Según el estudio, la autoestima no representa un factor importante en el rendimiento académico de los estudiantes, ya que mayoría concretamente el $65 \%$, demuestran una autoestima muy alta. Concluir que es indiferente de las calificaciones obtenidas, los que consideran que lo han hecho mejor de lo que han sido evaluados. No se puede decir lo mismo con los hábitos de estudio. La mayoría emplea malos hábitos de estudio, estudian la noche anterior un 29,4\%, no dedican suficiente tiempo para preparar el material, no suelen realizar mapas conceptuales de las materias, etc. La organización del tiempo también resulta ser un factor imprescindible y está ligado a los hábitos de estudio. La motivación se pierde cuando los estudiantes superan el límite de ansiedad. En conclusión, es imprescindible que en primer año universitario los estudiantes pasen un curso de orientación en la universidad. Dicho curso debería incluir métodos y técnicas de estudio, organización del tiempo, etc. que les permita al alumnado optimizar el tiempo de estudio y obtener mejor rendimiento académico.

\section{Referencias}

Choi, N. (2005). Self-efficacy and self-concept as predictors of college students' academic performance. Psychology in the Schools, 42(2), 197-205.

Falchikov N. and D. Boud (1989) "Student Self-Assessment in Higher Education: a Meta Analysis", Review of Educational Research, 59, 395-430

Green J., Nelson G., Martin A.J. \& Marsh H. (2006). The causal ordering of self-concept and academic motivation and its effect on academic achievement. International EDUCATION journal IEJ, 7( 4).

Guay, F., Marsh, H. W., \& Boivin, M. (2003). Academic self-concept and academic achievement: Developmental perspectives on their causal ordering. Journal of Educational Psychology, 95, 124136. 
Estudio de la autopercepción del rendimiento académico de los estudiantes universitarios de primer curso

Joppke, C., \& Morawska, E. (2003). Toward assimilation and citizenship: immigration in liberal nations. New York: Palagrave MacMillan.

Lent, R.W., Brown, S.D., \& Gore, P.A. (1997). Discriminant and predictive validity of academic selfconcept, academic self-efficacy, and mathematics-specific self-efficacy. Journal of Counselling Psychology, 44:307-315.

Liu, W. C., Wang, C. K. J., \& Parkins, E. J. (2005). A longitudinal study of students' academic selfconcept in a streamed setting: The Singapore context. British Journal of Educational Psychology, 75, 567-586.

Marsh, H.W. (1990a). A multi-dimensional, hierarchical model of self-concept: theoretical and empirical justification. Educational Psychology Review, 2:77-172.

Marsh H. (1990). "Causal ordering of academic self-concept and academic achievement: A multiwave, longitudinal panel analysis" Journal of Educational Psychology, 82, 646-656

Muijs, R. D. (1997). Symposium: Self perception and performance predictors of academic achievement and academic self-concept: a longitudinal perspective. British Journal of Educational Psychology, 67, 263-277.

Thorpe A. M. Snell, S. Hoskins and J. Bryant (2007). False Uniqueness: the Self-Perception of New Entrants to Higher Education in the UK and it's Implication for Access - a pilot study. Higher Education Quarterly, 61, 3-22 